\title{
Integralidade da consulta de enfermagem em hipertensão arterial: discutindo possibilidades
}

Comprehensiveness of nursing consultation in hypertension: discussing possibilities Integralidad de la consulta de enfermería en la hipertensión: discutiendo posibilidades

Tarciso Feijó da Silva ${ }^{1}$

\section{Resumo}

A hipertensão arterial é o principal fator de risco para doença cardiovascular e produz grandes repercussões no usuário, família e sociedade quando não controlada. $\mathrm{O}$ enfermeiro como integrante da equipe de saúde da família é responsável pelo atendimento dos usuários nesta linha de cuidado e desenvolve ações específicas, dentre elas a consulta de enfermagem. O objetivo deste artigo foi identificar nas principais bases de dados como a consulta de enfermagem na linha de cuidado em hipertensão arterial é realizada e apresentar possibilidades para a integralidade da mesma na Atenção Primária. Foi possível observar com o estudo consulta de enfermagem pouco orientada para longitudinalidade do cuidado e frágil orientação para atendimento voltado somente para controle dos casos já diagnosticados. Desta forma, procuramos destacar possibilidades de atuação do profissional enfermeiro durante a consulta de enfermagem na linha de cuidado em hipertensão tendo como referência o caderno de atenção básica nº 37 do Ministério da Saúde.

Descritores: Enfermagem, Atenção Primária à Saúde, Hipertensão

\begin{abstract}
Hypertension is a major risk factor for cardiovascular disease and produces great impact on the user, family and society if not controlled. The nurse as a member of the family health team is responsible for the service users this type of care and develop specific actions, among them the nursing consultation. The objective of this paper was to identify the main databases such as the nursing consultation on in hypertension care line is made and present possibilities for completeness thereof in Primary Care. It was observed with little study oriented nursing consultation to care longitudinality fragile and guidance for service only facing control cases already diagnosed. Thus, we seek to highlight the professional work opportunities nurse during the nursing consultation on care online hypertension with reference to the primary care notebook n. 37 of the Ministry of Health.
\end{abstract}

Key-words: Nursing, Primary Health Care, Hypertension

\section{Resumen}

La hipertensión es un importante factor de riesgo para la enfermedad cardiovascular y produce un gran impacto en el usuario, la familia y la sociedad si no se controla. La enfermera como miembro del equipo de salud de la familia es responsable de los usuarios de servicios de este tipo de atención y desarrollar acciones específicas, entre ellas la consulta de enfermería. El objetivo de este trabajo fue identificar las principales bases de datos tales como la consulta de enfermería en el cuidado de la hipertensión en línea se hace y las posibilidades actuales para la totalidad de ellas en atención primaria. Se observó con poco estudio orientado a la consulta de enfermería para cuidar

\footnotetext{
1 Mestre em Saúde Pública pela Escola Nacional de Saúde Pública (ENSP). Enfermeiro. Policlínica Piquet Carneiro/UERJ. Av. Marechal Rondon, no 381, Bairro: São Francisco Xavier, Cidade: Rio de Janeiro-RJ, Brasil. CEP: 20950-003. email: tarcisofeijo@yahoo.com.br
} 
ISSN 2179-6750

longitudinalidad frágil y orientación sólo para servicio de control frente a los casos ya diagnosticados. Por lo tanto, buscamos resaltar la oportunidades de trabajo profesional de la enfermera en la consulta de enfermería sobre la hipertensión en línea de atención en relación con el bloc de notas el apartado de atención primaria n. 37 del Ministerio de Salud.

Palabras-claves: Enfermería, Atención Primaria de Salud, Hipertensión

\section{Introdução}

A Estratégia de Saúde da Família (ESF) é vista como essencial para reorganização dos serviços de saúde no Brasil ${ }^{1}$. É entendida como a porta de entrada preferencial do sistema de saúde e está inserida em uma rede de atenção à saúde tendo por finalidade oferecer o primeiro contato às pessoas quando procuram atendimento ${ }^{2}$. Responde pela ordenação e coordenação do cuidado e como principal acesso dos usuários aos serviços de saúde. No Sistema Único de Saúde é o ponto responsável por atender a maior parte dos problemas de saúde dos usuários.

A ESF é composta por médicos, enfermeiros, técnicos ou auxiliares de enfermagem e agentes comunitários saúde. Estes desenvolvem diferentes ações, dentre elas o acompanhamento dos usuários com hipertensão arterial. A consulta nesta linha de cuidado é atribuição privativa dos profissionais médicos e enfermeiros ${ }^{3}$ sendo essencial para longitudinalidade do cuidado e melhoria da qualidade de vida dos usuários assistidos.

A consulta de enfermagem nas diferentes linhas de cuidado é tida como importante estratégia para o cuidado e o enfermeiro precisa conhecer os diferentes processos que a envolvem. No Brasil, a Política Nacional de Atenção Básica (PNAB), aponta a necessidade de utilização de protocolos ou outras normativas técnicas estabelecidas pelo gestor federal, estadual, municipal ou do Distrito Federal e das disposições legais da profissão na realização da consulta de enfermagem pelo profissional enfermeiro ${ }^{3}$. O Ministério da Saúde (MS) normatiza o acompanhamento de determinadas condições através de publicações e estas contribuem para o cuidado sistemático dos usuários do Sistema Único de Saúde. Uma das publicações mais relevantes está relacionada às doenças crônicas não transmissíveis - Hipertensão arterial (HAS) e Diabetes Mellitus (DM) - que são condições alvo para atuação dos profissionais da ESF, tendo em vista que estas doenças representam dois dos principais fatores de risco para as doenças cardiovasculares e constituem a principal causa de morbimortalidade na população brasileira. A hipertensão arterial afeta de 11 a $20 \%$ da população adulta com mais de 20 anos. Cerca de $85 \%$ dos pacientes com acidente vascular encefálico (AVE) e 40\% das vítimas de infarto do miocárdio apresentam hipertensão associada. O diabetes, por sua vez, atinge a gestante nas diferentes faixas etárias, sem qualquer distinção de raça, sexo ou condições sócio-econômicas. Na população adulta, a prevalência desta doença é de 7,6\%. 
ISSN 2179-6750

As duas doenças levam, com frequência, à invalidez parcial ou total do indivíduo, com graves repercussões para o paciente, sua família e a sociedade ${ }^{4}$.

Nesse contexto, a consulta de enfermagem aparece como sendo importante, na medida que contribui para o diagnóstico precoce de novos casos de hipertensão arterial e diabetes mellitus e para acompanhamento e controle dos casos antigos.

O objetivo deste artigo, considerando o exposto, é verificar de acordo com estudos já realizados como a consulta de enfermagem na linha de cuidado em hipertensão arterial tem sido realizada e apresentar possibilidades para a integralidade da mesma na Atenção Primária.

\section{Metodologia}

Este estudo de abordagem qualitativa ${ }^{5}$ analisou a produção relacionada à consulta de enfermagem na linha de cuidado em hipertensão arterial entre os anos de 2004 e 2015. A pesquisa foi realizada na base de dados da Medline e Bdenf com a utilização dos descritores "Enfermagem", “Atenção Primária à Saúde” e "Hipertensão". Emergiram 10 artigos completos em português que foram analisados visando identificar a forma como as consultas de enfermagem na linha de cuidado em hipertensão eram realizadas. Os achados da análise das produções permitiu à luz do caderno de atenção básica $n^{\circ} 37$ - Hipertensão arterial sistêmica - principal instrumento que orienta a consulta de enfermagem em hipertensão arterial apresentar possibilidades para integralidade da consulta de enfermagem nesta linha de cuidado.

\section{Resultados}

Dentre os artigos encontrados 02 avaliavam a prática do enfermeiro relacionada à consulta de enfermagem considerando a necessidade de integralidade do cuidado, longitudinalidade e tratamento da HAS de forma individualizada, com enfoque para educação em saúde, modificações dos hábitos de vida e, se necessário, medicamentos ${ }^{6}$. De acordo com os mesmos a consulta de enfermagem em HAS deve considerar o perfil epidemiológico da população, como ferramenta para cuidado individualizado e para planejamento de ações que mobilize o autocuidado e a corresponsabilidade dos usuários, deve ainda considerar os grupos de risco e os usuários com pressão arterial limítrofes e predispostos a hipertensão arterial; a necessidade de estratificação de risco para doenças cardiovasculares e o acompanhamento propriamente dito do indivíduo com HAS. Ainda, de que no acompanhamento para os grupos de risco e indivíduos com Pressão arterial (PA) limítrofes, deve ser preconizado o incentivo a mudança no estilo de vida (MEV) para adoção de hábitos saudáveis e prevenção primária da $\mathrm{HAS}^{7}$. 
ISSN 2179-6750

Os demais artigos analisados, por sua vez, tratavam especificamente dos motivos que levavam o usuário a procurar atendimento, de ações grupais voltadas para atendimento dos usuários hipertensos, dos hábitos alimentares e sua relação com a hipertensão e especificamente dos fatores de risco para cardiopatia em idosos. Não havia discussão específica que envolvesse a importância da consulta de enfermagem, nem relação estabelecida no debate com o que está preconizado pelo Ministério da Saúde. Nenhum destes artigos possibilitou observar valorização da consulta de enfermagem como prática que possibilita prevenção precoce da hipertensão arterial e controle adequado dos casos já diagnosticados.

\section{Discussão}

O Ministério da Saúde estabelece que o foco do processo educativo deve ser voltado, para orientação daquelas medidas que comprovadamente reduzem a pressão arterial, entre elas hábitos alimentares adequados para manutenção do peso corporal e de um perfil lipídico desejável, estímulo à vida ativa e aos exercícios físicos regulares, redução da ingestão de sódio, redução do consumo de bebidas alcoólicas, redução do estresse e abandono do tabagismo ${ }^{8}$. Existem evidências do efeito dessas medidas na redução da pressão arterial, que além do mais possuem baixo custo, ajudam no controle de fatores de risco para outros agravos, aumentam a eficácia do tratamento medicamentoso (necessitando de menores doses e de menor número de fármacos) e reduzem o risco cardiovascular 9 . Para acompanhar o impacto das mudanças no estilo de vida dos indivíduos com pressão arterial limítrofe o Ministério da Saúde traz algumas recomendações e relaciona a redução esperada da pressão arterial para cada uma delas que devem ser acompanhadas de forma sistemática através das consultas de enfermagem.

$\mathrm{Na}$ consulta de enfermagem na linha de cuidado em hipertensão arterial com foco na estratificação de risco para doenças cardiovasculares a recomendação é a utilização do Escore de Framingham que tem como objetivo estimar o risco de cada indivíduo de sofrer uma doença arterial coronariana nos próximos dez anos. Este, considera a presença de múltiplos fatores de risco, como sexo, idade, níveis pressóricos, tabagismo, níveis de HDLc e LDLc. A partir da estratificação de risco, selecionam-se indivíduos com maior probabilidade de complicações, os quais se beneficiarão de intervenções mais intensas. $O$ processo de estratificação segue 03 etapas que precisam ser abordadas na consulta de enfermagem para garantia da integralidade do cuidado e melhoria da qualidade de vida dos usuários com hipertensão. A primeira etapa segue os achados do exame clínico e anamnese indicativos de risco para Doença Cardiovascular (RCV).

Se, o usuário, apresenta apenas um fator de risco baixo/intermediário, não há necessidade de 118 
ISSN 2179-6750

calcular o escore, pois ele é considerado como baixo RCV. Se, apresenta, ao menos um fator de alto $\mathrm{RCV}$, também não há necessidade de calcular o escore, pois esse paciente já é considerado como alto RCV. O cálculo deve ser realizado somente quando o usuário apresentar mais de um fator de risco baixo/intermediário. A avaliação da idade, exames de LDLc, HDLc, pressão arterial e tabagismo são componentes da segunda etapa. A terceira etapa termina com o estabelecimento de uma pontuação que define o risco percentual de evento cardiovascular em dez anos para homens e mulheres e classifica os indivíduos permitindo acompanhamento segundo o risco identificado. O seguimento dos indivíduos considerados como de baixo risco, risco intermediário e alto risco devem acontecer respectivamente anualmente após orientação relacionada ao estilo de vida saudável; semestralmente após orientações sobre estilo de vida saudável e, se disponível na UBS ou comunidade e se desejo da pessoa, encaminhamento para ações coletivas de educação em Saúde; e trimestralmente após orientações sobre estilo de vida saudável e, se disponível na UBS ou comunidade e, se desejo da pessoa, encaminhamento para ações de educação em saúde coletiva ${ }^{9}$. A estratificação de risco por sua vez, deve ser realizada sempre que possível nas consultas de enfermagem, tendo em vista que o risco pode diminuir ou aumentar de acordo com adesão ao tratamento.

A consulta de enfermagem para acompanhamento da HAS já diagnosticada pressupõe aplicação da sistematização da assistência de enfermagem (SAE) orientada segundo Resolução do COFEN $n^{\circ} 358$, de 15 de outubro de $2009^{10}$ e deve ter como foco os fatores de risco que influenciam o controle da hipertensão, a possibilidade de prevenção secundária e manutenção de níveis pressóricos abaixo da meta. Assim, foi possível observar nos artigos analisados profunda preocupação em garantir atendimento/acesso dos usuários hipertensos aos serviços de saúde através de atividades individuais e coletivas, e estas de forma isolada, sem considerar a normatização específica e as políticas do Ministério da Saúde relacionada à HAS demonstraram-se frágeis para prevenir o aparecimento da doença, controlar os casos existentes e diminuir a morbimortalidade por doenças cardiovasculares causadas pela HAS.

\section{Conclusão}

O enfermeiro na ESF assume diferentes papeis no acompanhamento e controle da hipertensão arterial e possui autonomia para direcionamento de ações e serviços de saúde voltados para prevenção, promoção e reabilitação da saúde das pessoas sob sua responsabilidade. A consulta de enfermagem em HAS é ação essencial considerando o contexto atual de envelhecimento da 
ISSN 2179-6750

população, aumento da expectativa de vida e prevalência dos fatores de risco (sedentarismo, tabagismo, estresse) que contribuem para recrudescimento das doenças e aumento da mortalidade por doenças crônicas não transmissíveis. Assim destacamos diferentes possibilidades de atuação do profissional enfermeiro, dentre elas grupos de promoção em saúde, salas de espera, discussão de casos em reunião de equipe e com a equipe do Núcleo de Apoio à saúde da família. No entanto, as consultas de enfermagem são de extrema importância e devem está voltadas para avaliação e orientação relacionada à mudança no estilo de vida dos usuários com fatores de risco e com pressão arterial limítrofe e para estratificação do risco e acompanhamento contínuo com enfoque para adesão e corresponsabilização dos usuários.

\section{Referências}

1. Brasil. Ministério da Saúde. Portaria no 2.488, de 21 de outubro de 2011. Aprova a Política Nacional de Atenção Básica, estabelecendo a revisão de diretrizes e normas para a organização da Atenção Básica, para a Estratégia Saúde da Família (ESF) e o Programa de Agentes Comunitários de Saúde (PACS). Brasília: Ministério da Saúde, 2011.

2. SMSDC. Superintendência de Atenção Primária. Guia de Referência Rápida. Carteira de Serviços: Relação de serviços prestados na Atenção Primária à Saúde / Secretaria Municipal de Saúde e Defesa Civil. Superintendência de Atenção Primária. - Rio de Janeiro: SMSDC, 2011. 128p.

3. Brasil. Ministério da Saúde. Secretaria de Atenção à Saúde. Departamento de Atenção Básica. Política Nacional de Atenção Básica / Ministério da Saúde. Secretaria de Atenção à Saúde. Departamento de Atenção Básica. - Brasília : Ministério da Saúde, 2012.

4. Brasil. Ministério da Saúde. Departamento de Atenção Básica. Área Técnica de Diabetes e Hipertensão Arterial. Hipertensão arterial sistêmica (HAS) e Diabetes Mellitus (DM): protocolo / Ministério da Saúde, Departamento de Atenção Básica. Área Técnica de Diabetes e Hipertensão Arterial. - Brasília: Ministério da Saúde, 2001.

5. Minayo MC. Desafio do Conhecimento: pesquisa qualitativa em saúde. $10^{a}$ ed. São Paulo: HUCITEC, 2007.

6. Brasil. Ministério da Saúde. Secretaria de Atenção à Saúde. Departamento de Atenção Básica. Diretrizes para o cuidado das pessoas com doenças crônicas nas redes de atenção à saúde e nas linhas de cuidado prioritárias / Ministério da Saúde, Secretaria de Atenção à Saúde, Departamento de Atenção Básica. - Brasília: Ministério da Saúde, 2013.

7. Brasil. Ministério da Saúde. Secretaria de Atenção à Saúde. Departamento de Atenção 120 
ISSN 2179-6750

Básica. Estratégias para o cuidado da pessoa com doença crônica: hipertensão arterial sistêmica / Ministério da Saúde, Secretaria de Atenção à Saúde, Departamento de Atenção Básica. - Brasília: Ministério da Saúde, 2013.

8. Chobanian AV. (Org). The seven the report of the Joint National Committee on Prevention, Detection, Evaluation, and Treatment of High Blood Pressure.Washington D.C.: National Institute sofh ealth: 2004.

9. Sega R. et al. Prognostic value of ambulatory and home blood pressures compared with office blood pressure in the general population: follow-up results from the Pressioni Arteriose Monitorate e Loro Associazioni (PAMELA) study. Circulation, Baltimore, v. 111, n. 14, p. 1777-1783, apr. 2005.

10. COFEN. Conselho Federal de Enfermagem. Resolução COFEN/1993. 